RADIOLOGIC VIGNETTE NO. 2

\title{
THE DIAGNOSTIC VALUE OF BUTTRESSING OF THE FEMORAL NECK
}

\author{
WILLIAM MARTEL and ETHAN M. BRAUNSTEIN
}

The skeletal system can react to disease in limited ways and it is therefore not surprising that different pathologic conditions may to some extent exhibit similar radiologic features. Certain combinations of findings are more likely to occur in some diseases than others. One may speak of the "predictive" value of various radiologic features relative to the clinical condition being considered.

Buttressing of the medial aspect of the femoral neck is much more common in some diseases of the hip than others. Figures 1 to 4 illustrate the roentgenographic findings in the hip in four joint diseases. Buttressing of the femoral neck is present in Figures 1 and 2, but absent in Figures 3 and 4 . In addition there are other radiologic features that are important considerations in the differential diagnosis. The four diseases represented are: ischemic osteonecrosis, rheumatoid arthritis, ankylosing spondylitis, and osteoarthritis. Can you match these diseases with the figures? The answer may be found at the end of the article.

Buttressing, or subperiosteal bone apposition, of the femoral neck is frequent in osteoarthritis and ischemic necrosis of the femoral head. It was evident radiologically in 33 of 73 consecutive patients with idiopathic osteoarthritis (1) and in 24 of 32 hips in which

William Martel, M.D.: Professor of Radiology, University of Michigan, Ann Arbor, Michigan; Ethan M. Braunstein, M.D.: Instructor of Radiology, University of Michigan, Ann Arbor, Michigan. Address reprint requests to: William Martel, M.D., Department of Radiology, University Hospital, Ann Arbor, Michigan 48109. there was ischemic necrosis of the femoral head associated with various conditions (2). It has also been described in congenital subluxation of the hip (3). However, it is relatively uncommon in rheumatoid arthritis and ankylosing spondylitis and, when it does occur in these diseases, it is usually minimal. Recent review of our case material (4) showed that it occurred in 4 of 45 rheumatoid hips (26 patients) and was equivocal in 1 other. Similarly, it was detected in only 1 of 49 hips in 29 patients with ankylosing spondylitis (Figure 5) and was equivocal in 2 others. Its occurrence in these diseases is apparently not related to the severity of joint involvement.

Buttressing is thought to be secondary to changes in mechanical stress that follow alterations in weightbearing at the joint surface (3). In these cases more weight is presumably borne by the medial aspect of the femoral neck. Presumably, repeated microfractures give rise to focal bone apposition at this site. The observation that patients with osteoarthritis and buttressing also often exhibit an accentuation of vertical trabeculae that extend from the medial femoral cortex superiorly to the joint surface (Figure 1) supports this concept. The mechanism is probably the same in ischemic necrosis. A microfracture of the medial femoral neck was observed radiologically in 1 patient with ischemic necrosis of the femoral head (2), suggesting that the mechanism of buttressing in this disease is probably the same.

Lloyd-Roberts (1) has suggested that buttressing is caused by elevation of the periosteum as a result of 


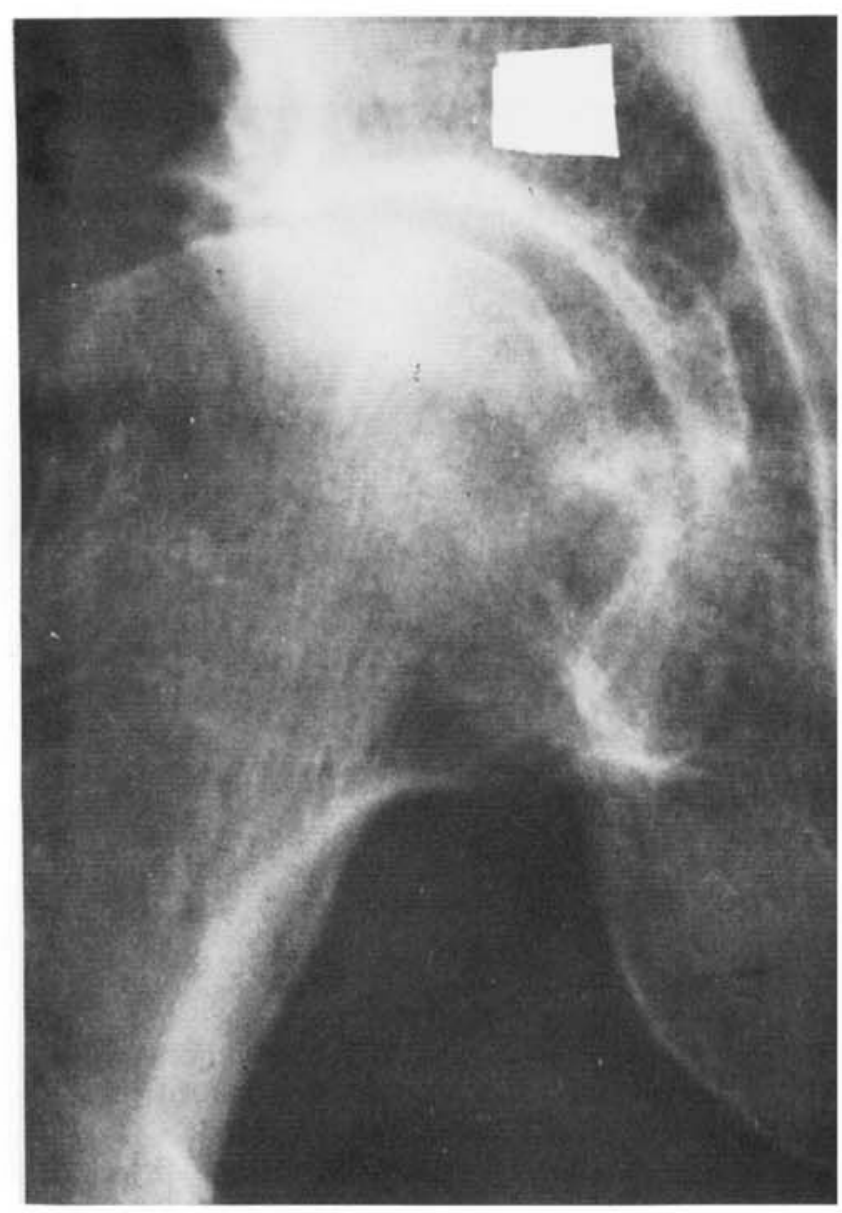

Figure 1.

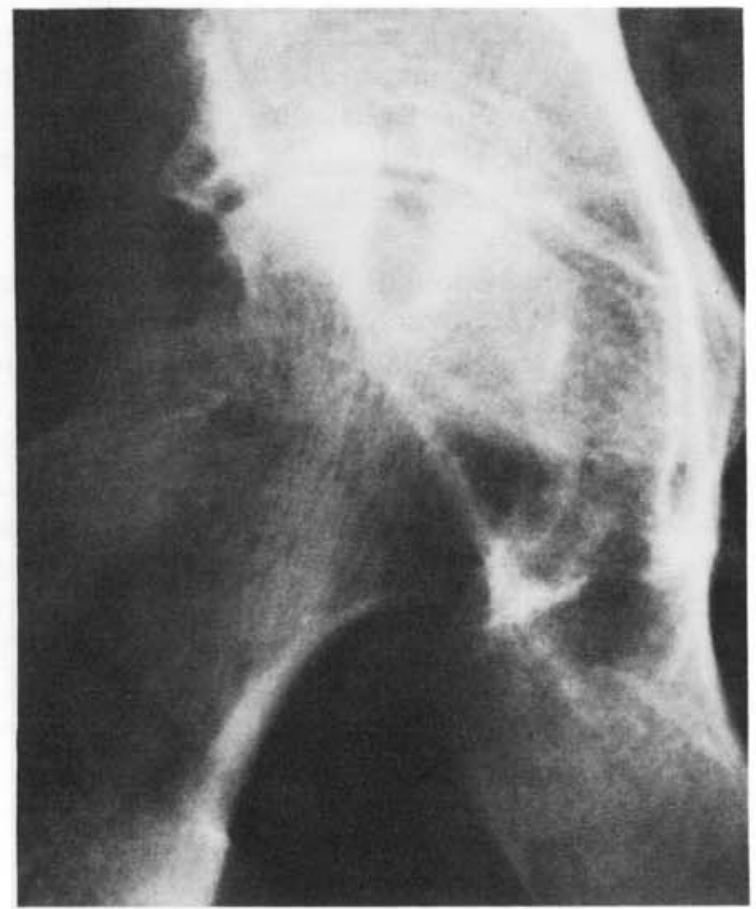

Figure 3.

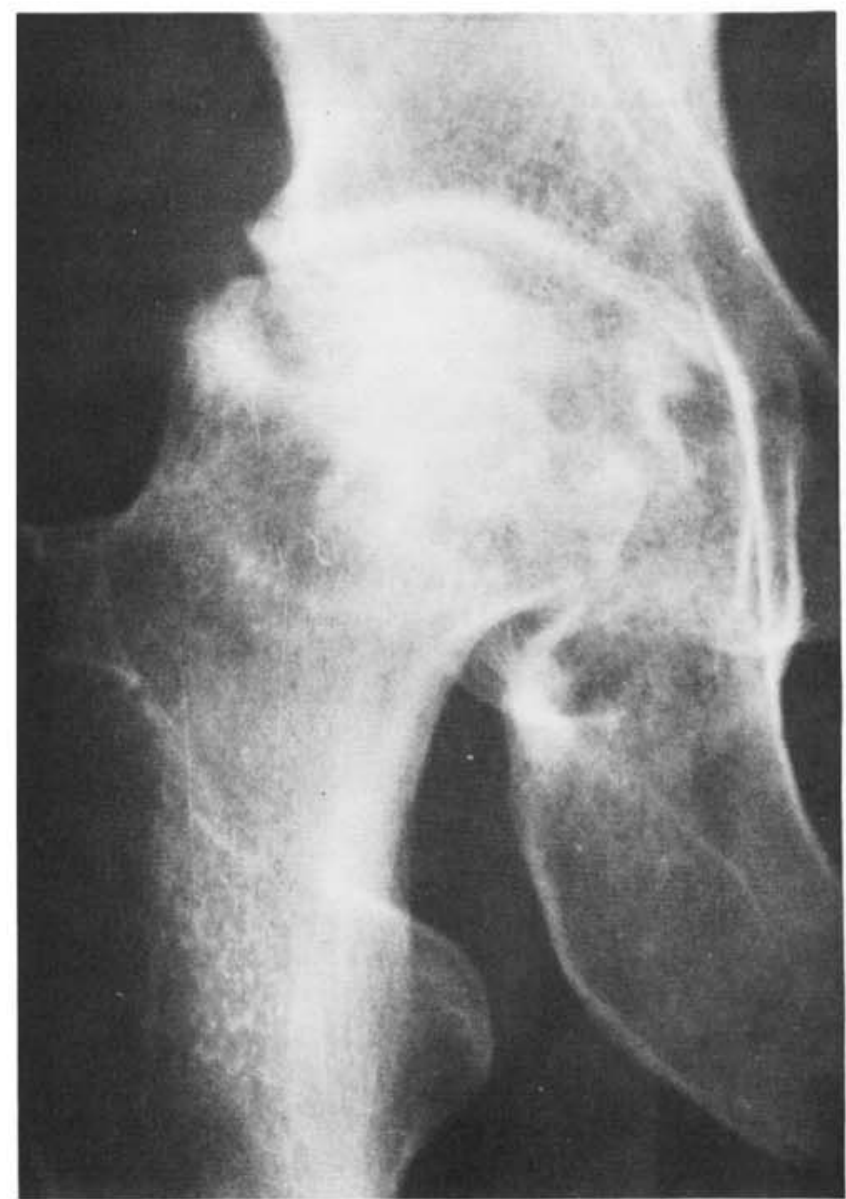

Figure 2.

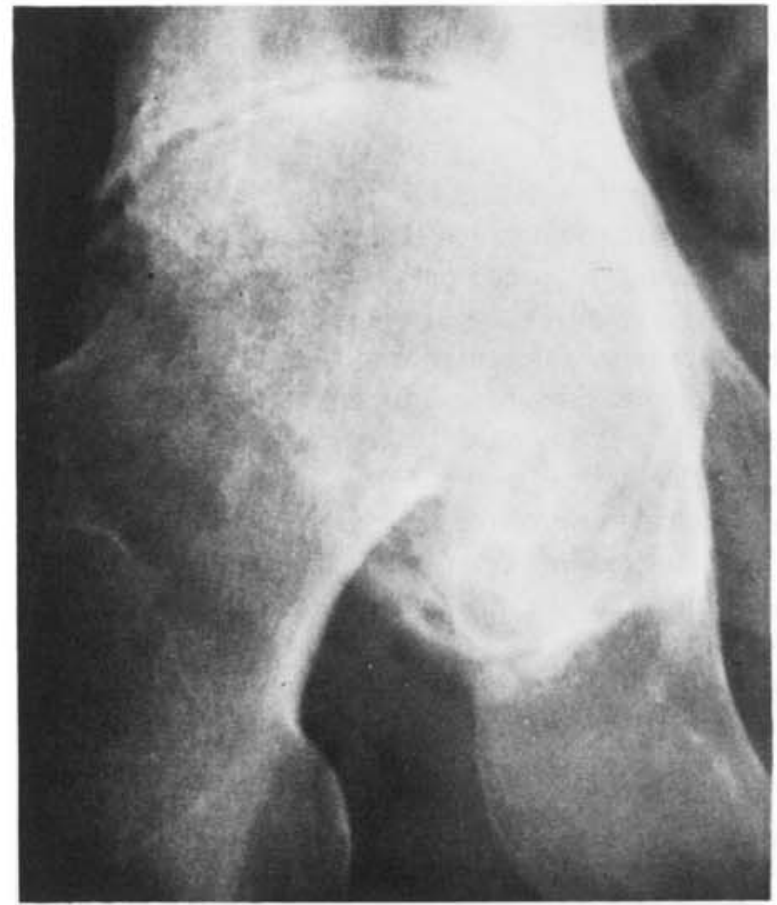

Figure 4. 
traction transmitted through the posteroinferior capsular reflection. He noted that the joint capsule is characteristically fibrotic and foreshortened and is often fused to the periosteum in this region. Consequently, he believed that buttressing is more likely in osteoarthritis when subluxation is also present. This may indeed be a factor in some cases.

It seems reasonable to postulate that buttressing is relatively uncommon in rheumatoid arthritis and ankylosing spondylitis because alterations of the joint surface in these diseases do not give rise to increased localized loading on the femoral head. Unlike the situation in osteoarthritis, there is uniform involvement of the articular cartilage with destruction taking place both medially and superiorly. To be sure, this causes abnormal mechanical stress on the subchondral cortex but it is not localized to one area and the force is not as apt to be selectively transmitted to the medial femoral neck. In

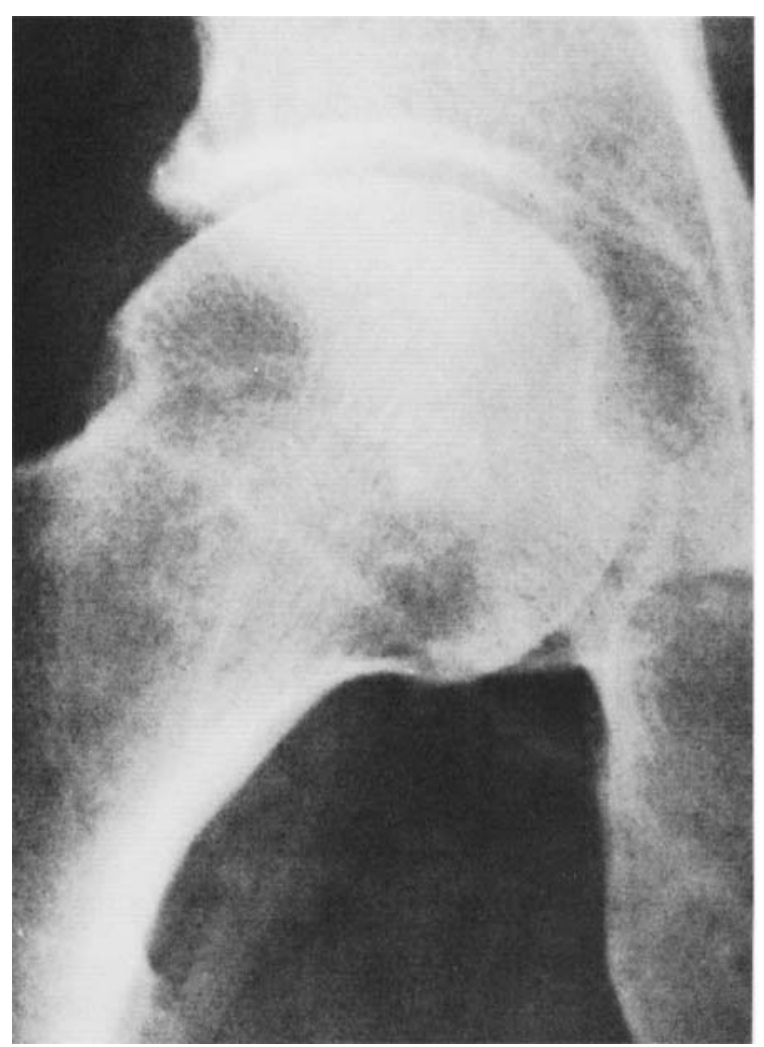

Figure 5. Moderate buttressing in ankylosing spondylitis. The cartilage space is minimally diminished. There is an irregularity of the lateral chondro-osseous junction suggesting prior surface erosion and bone apposition. The sacroiliac joints were fused and the symphysis pubis and ischial margins were affected. The opposite hip was similarly involved but there was no buttress. fact, there is medial migration of the femoral head in these diseases and acetabular protrusion is often present, a finding that indicates that stress on the bone is transmitted to the medial acetabular wall. It should not be surprising that buttressing occasionally occurs in these diseases inasmuch as destruction of the articular cartilage does lead to abnormal stress on the femoral head which is partially transmitted to the medial femoral cortex.

Hip involvement in these diseases is generally associated with bone atrophy; this may explain why the periosteal reaction is typically minimal to moderate and almost never exuberant. It should be noted that acetabular protrusion does not preclude buttressing. We recently observed a patient with rheumatoid arthritis and acetabular protrusion in whom moderate buttressing was also present (Figure 6).

Typical features of osteoarthritis are exhibited in

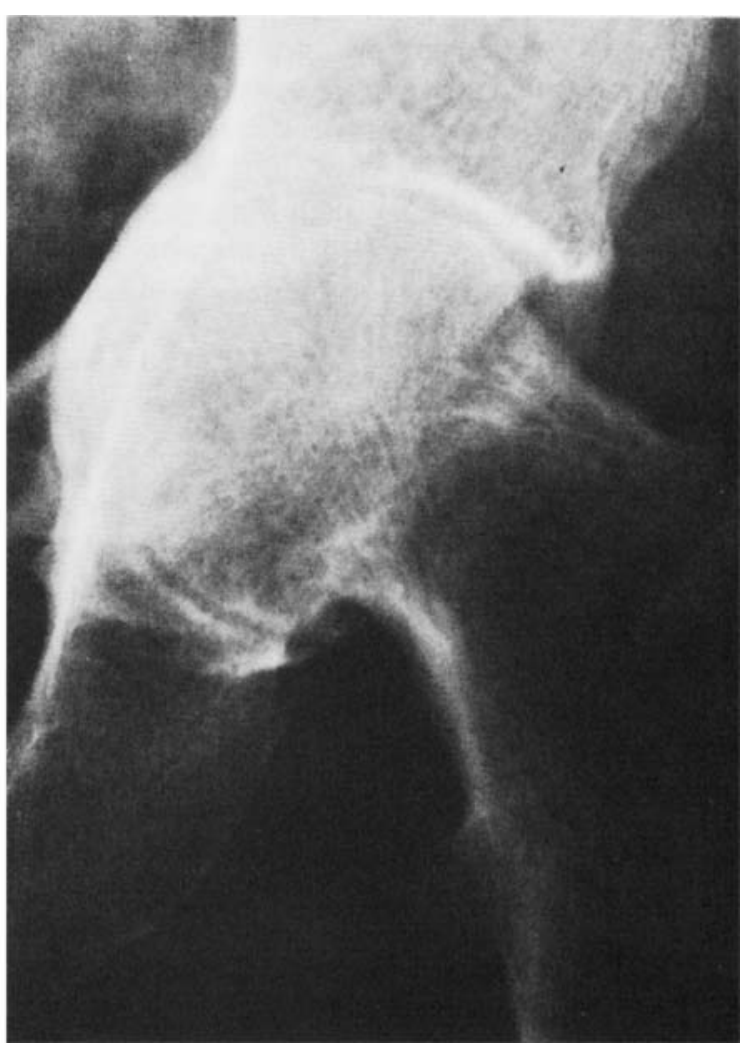

Figure 6. Rheumatoid arthritis with acetabular protrusion and moderate buttressing. The cartilage space is reduced throughout and osteophytes are absent. Note the sclerosis and bone apposition on the medial acetabular margin and the coarsening of the trabeculae that extend vertically from the medial aspect of the femoral neck. The minimal irregular bone apposition at the superior aspect of the junction of femoral head and neck suggests that this too was the site of stress fracture. 
Figure 1. These include nonuniform narrowing of the cartilage space and sclerosis of the subchondral bone superiorly where the interosseous space is reduced. Osteophyte formation is a conspicuous feature that characteristically appears as a ring of bone on the femoral head near the edge of the articular cartilage and at the acetabular margins. The margins appear as a "double acetabular contour" and is responsible for lateral displacement of the femoral head. In osteoarthritis the sclerosis of the femoral head causes an accentuation and sharpening of the subchondral bone margins but in osteonecrosis, the sclerosis is characteristically associated with an indistinctness and blurring of these margins. Such blurring may be an early clue to the diagnosis. Furthermore, the sclerosis in osteoarthrit is is usually limited to the upper part of the femoral head, whereas in osteonecrosis it often involves much of the femoral head, sometimes extending into the upper neck as well. Osteophyte formation is not a feature of ischemic osteonecrosis. (Compare Figures 1 and 2.)

Cartilage space narrowing is typically uniform in both rheumatoid arthritis and ankylosing spondylitis. Parenthetically, this is not specific for these diseases inasmuch as uniform narrowing is characteristic of other inflammatory diseases, including infectious arthritis. In addition rheumatoid arthritis is characterized by a relative absence of bone production, even at sites of extensive bone erosion (Figure 3). However, in ankylosing spondylitis the osteitis and bone erosion are usually associated with sclerosis and bone apposition (Figure 4). The bone apposition should not be confused with osteophytes. In both diseases marginal erosions are usually first evident in the femoral head at the chondro-osseous junction, particularly laterally. These erosions tend to be superficial and subtle in ankylosing spondylitis, whereas in rheumatoid arthritis there is often extensive bone destruction. Large osteolytic lesions such as those seen in Figure 3 are distinctly unusual in ankylosing spondylitis.

Answer: Figure 1, osteoarthritis; Figure 2, ischemic necrosis; Figure 3, rheumatoid arthritis, Figure 4, ankylosing spondylitis.

\section{REFERENCES}

1. Lloyd-Roberts GC: The role of capsular changes in osteoarthritis of the hip joint. J Bone Joint Surg 35B:627-642 1953

2. Martel W, Sitterley, BH: Roentgenologic manifestations of osteonecrosis. Am J Roentgenol 106:509-522, 1969

3. Wiberg G: Studies on dysplastic acetabula and congenital subluxation of the hip joint. Acta Chir Scand (suppl) 58:87, 1939

4. Martel W, Braunstein E: Unpublished data 